\title{
Removal of central obscuration and spider arm effects with beam-shaping coronagraphy
}

\author{
L. Abe ${ }^{1}$, N. Murakami ${ }^{2}$, J. Nishikawa ${ }^{2}$, and M. Tamura ${ }^{1}$ \\ 1 Optical and Infrared Division and Extrasolar Planet Project Office, National Astronomical Observatory of Japan, Ōsawa 2-21-1, Mitaka-city, \\ 181-8588 Tōkyō, Japan \\ e-mail: abe@optik.mtk.nao.ac.jp \\ 2 MIRA project, National Astronomical Observatory of Japan, Ōsawa 2-21-1, Mitaka-city, 181-8588 Tōkyō, Japan
}

Received 5 August 2005 / Accepted 16 November 2005

\section{ABSTRACT}

This paper describes a method for removing the effect of a centrally obscured aperture with additional spider arms in arbitrary geometrical configurations. The proposed method is based on a two-stage process where the light beam is first shaped to remove the central obscuration and spider arms, in order to feed a second, highly efficient coronagraph. The beam-shaping stage is a combination of a diffraction mask in the first focal plane and a complex amplitude filter located in the conjugate pupil. This paper specifically describes the case of using Lyot occulting masks and circular phase-shifting masks as diffracting components. The basic principle of the method is given along with an analytical description and numerical simulations. Substantial improvement in the performance of high-contrast coronagraphs can be obtained with this method, even if the beam-shaping filter is not perfectly manufactured.

Key words. instrumentation: high angular resolution - telescopes - techniques: miscellaneous

\section{Introduction}

High contrast or high dynamic range imaging techniques with coronagraphs are now envisaged for current and future direct detection and characterization of extrasolar planets. The term coronagraph encompasses several kinds of methods, namely, focal mask coronagraphs (e.g. Lyot 1939; Roddier \& Roddier 1997; Rouan et al. 2000; Kuchner \& Traub 2002), interferometric coronagraphs (e.g. Gay \& Rabbia 1997; Tavrov et al. 2002), shaped pupil apodizers (e.g. Spergel et al. 2001; Kasdin et al. 2003) and amplitude apodizers (e.g. Guyon 2003).

Even if many variants of these techniques have been proposed (e.g. Abe et al. 2001; Baba \& Murakami 2003; Soummer et al. 2003), only a few of them have been successfully tested in laboratory. Finally only an even smaller number has been tested on the sky for real scientific observations. There are mainly two reasons. First, the ground-based observatories need very high performance adaptive optics systems in order to obtain significant high-contrast performance. Second, most of the existing telescopes are not optimized for these new coronagraphs. In order to reach very high contrast $\left(>10^{6}\right)$, many coronagraphic concepts require a clear, unobscured, and spider-arm-free pupil geometry. But if some of theses coronagraphs are not sensitive to the central obscuration (CO hereafter), almost all of them (except AIC and apodized Roddier coronagraphs) suffer from the presence of the secondary mirror spider arms (SA hereafter). Some means can be found to overcome these geometry problems, but in all cases it puts strong constraints on the telescope's optical design, such as a small secondary mirror and very thin spiders (e.g. Vanderbei et al. 2003; Vanderbei et al. 2004, for shaped pupils). Therefore, it is often stated that optimized coronagraphic telescopes should preferably be off-axis (e.g. Kuhn \& Hawley 1999).

Unfortunately none of the existing instruments is really optimized in that way for high performance coronagraphy. The solution of using an off-axis sub-pupil of an existing large telescope sounds too shocking to be envisaged realistically. Even space missions with coronagraphic capabilities (e.g. HST, JWST or SPICA) are far from being optimized for very high contrast purposes. One of the reasons is that a compromise must be found between several onboard instrument requirements. Moreover, the coronagraphic mode often have a lower priority in these missions. Under these conditions we must either find new types of coronagraphs (the hard way), modify these telescopes (an impossible mission), or try to find techniques to overcome the pupil geometry problem, which is the point of this paper.

Our proposed method is based on a two-stage process where the light beam is firstly shaped to remove the $\mathrm{CO}$ and SA in order to match a second higher efficiency coronagraph. In Sect. 2 we describe the approach of the possible beam-shaping 
technique, and put forward the basis of the method. In Sect. 3, we give the general equations needed to obtain the desired beam-shaper and discuss intrinsic constraints. Section 4 illustrates the concept by the use of simple Lyot occulting masks and circular phase-shifting masks as first diffracting elements. In Sect. 5 we examine the effect of possible defects in the beamshaping filter component and its consequences on a full coronagraphic set-up. We finally discuss some other technical implementation issues such as chromatism.

\section{Approach}

The basic idea is to reshape the light beam in order to fill the gaps created by the CO and SA. Similar approaches have already been considered by the use of generic "pupil densification" techniques (Labeyrie 1999). They consist in re-mapping the different sectors of the pupil (delimited by the SA) as a way of removing both the CO and SA. Aime et al. (2003a) analyze a direct densification that results in a complete removal of the $\mathrm{CO}$ and SA geometry, but leads to a non-linear deformation of the focal plane image for off-axis objects. In addition, this method is also largely constrained by the size of the CO.

More recent works based on the two-mirror apodization technique (TMA, or beam reshaping - Maylak (1992) for laser beam reshaping, Guyon (2003) and Traub \& Vanderbei (2003) for coronagraphy) was proposed either for a purely apodizing purpose (Guyon 2003) or in combination with the four quadrant phase mask (FQPM hereafter) to minimize the effects of the CO alone (Murakami \& Baba (2005)). However, a distortion of the off-axis images still occurs, which necessitates a similar de-mapping mirror combination. Guyon points out (private communication) that the removal of SA with the TMA should be very difficult to achieve in practice because of the highly aspherical surfaces needed.

These approaches are already in the "philosophy" of the multiple stage coronagraphs. This multi-stage approach was also recently considered for coronagraphy with a combination of a classical Lyot coronagraph and a shaped pupil mask (Chakraborty et al. 2005). Another way consists in combining nulling interferometry and coronagraphy (Nishikawa et al. 2005). The perfect example of multi-stage coronagraphy is, however, proposed by Aime \& Soummer (2003b) with the cascading Multi-Stage Prolate-(amplitude) $)^{1}$ Apodized Lyot Coronagraph (MS-PALC). It theoretically leads to an arbitrary high contrast since the extinction efficiency is a power of the number of stages involved. In practice, the efficiency of such a system will be limited to a few stages due to the increased number of optical surfaces and alignment difficulties. The properties of the prolate Lyot coronagraph are such that it also works with a CO (totally insensitive) at the expense of reducing each stage performance. Nevertheless, the multi-stage approach still works. The presence of SA somehow affects the MS-PALC properties, but the multi-stage effect still works within certain limits. The motivation for removing the

\footnotetext{
${ }^{1}$ To avoid confusion with the shaped pupil coronagraphs that also use prolate geometry to cancel the light in a given area of the focal plane.
}

internal pupil geometry is the possible use of a highly performing coronagraph in a multi-stage approach, while minimizing the number of stages: a first stage is used to remove the $\mathrm{CO}$ and SA, then a high performance coronagraph can be applied that suffers neither from CO nor SA.

It can easily be demonstrated that the complex amplitude in the coronagraphic pupil (i.e. after the diffracting effect of a focal mask in amplitude and/or phase) is written as the sum of two terms: the entrance pupil function $(\mathbf{P})$ and the part of the wave that is diffracted by the coronagraphic mask $(\mathbf{C})$ - see Aime et al. (2001). Generally speaking, the part $\mathbf{C}$ is a continuous function in phase and amplitude that spreads to infinity. Therefore the addition of these two components $\mathbf{P}$ and $\mathbf{C}$ results in a field (the geometrical pupil support) where coherent light is present everywhere, especially at the CO and SA location, in opposition to the entrance pupil alone. The idea here is to exploit this natural gap filling to produce a full, continuous pupil that can be used later for another coronagraphic stage.

In order to avoid confusion, the following sections will only deal with the first beam-shaping stage, which we also call coronagraphic stage because some light is rejected but with low efficiency. Therefore, the reference to the coronagraphic pupil will be the pupil where the beam is shaped and which corresponds to the amplitude distribution for a perfectly on-axis point source. We voluntarily neglect the second coronagraphic stage in Sects. 2-4, since after the beam is shaped, virtually any of the known coronagraphic concepts can be applied. In addition, we will also often refer to the unobscured part (of the pupil), which is the complementary pattern to the $\mathrm{CO}$ and SA (i.e. the effective mirror surface).

\section{Analysis}

Figure 1 is a schematic representation of the coronagraphic principle in focal mask coronagraphy. The focal-maskdiffracted part $\mathbf{C}$ "appears in negative amplitude on an image of the entrance-pupil" (Aime et al. 2001). We can therefore try to apply a complex amplitude filter $\mathbf{F}$ (modulus and phase) in this pupil plane in order to reshape the light beam and obtain a coherent continuous wavefront for the desired output pupil. The principle is depicted in Fig. 2. In the following, $\mathbf{F}$ refers to,

$\mathbf{F}=|\mathbf{F}| \times \mathrm{e}^{\mathrm{i} \varphi} \mathbf{F}$

and is defined only on the area limited by the outermost boundary of the geometric pupil (i.e. the full, unobscured pupil).

\subsection{General expression for the beam-shaping filter}

The coronagraphic pupil amplitude, after filter $\mathbf{F}$ is applied, can be expressed as,

$\mathbf{S B}=(\mathbf{P}+\mathbf{C}) \times \mathbf{F}$

where SB is the complex amplitude of the desired output beam that stands for shaped beam. Therefore, the complex amplitude filter modulus is,

$|\mathbf{F}|=\frac{|\mathbf{S B}|}{|\mathbf{P}+\mathbf{C}|}$ 


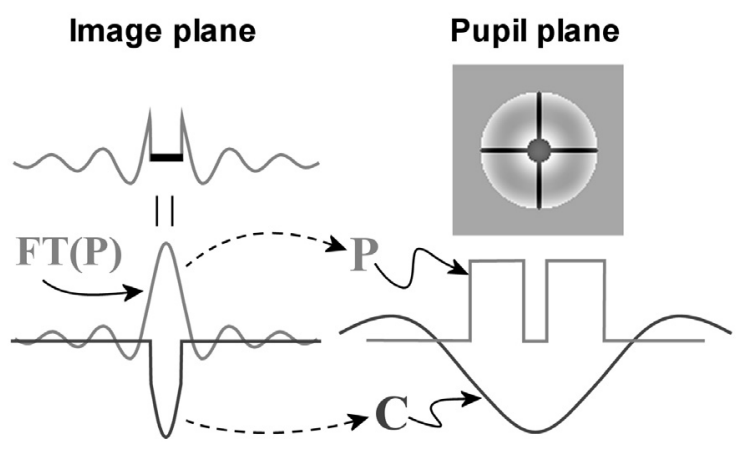

Fig. 1. The complex amplitude in the coronagraphic pupil plane is the sum of two terms: the entrance pupil function $(\mathbf{P})$ and the part of the wave that is diffracted by the coronagraphic mask $(\mathbf{C})$. In the coronagraphic pupil plane (top-right), the amplitude function exhibits the $\mathrm{CO}$ and SA patterns "in negative", but filled with coherent light. FT( $\cdot)$ denotes the Fourier transformation.

and in order to conserve the energy, we need to normalize this expression such that

$|\mathbf{F}|=\frac{1}{\max \left(\frac{|\mathbf{S B}|}{|\mathbf{P}+\mathbf{C}|}\right)} \frac{|\mathbf{S B}|}{|\mathbf{P}+\mathbf{C}|}$,

whereas the phase map is obtained by writing,

$\varphi_{\mathbf{F}}=\arg (\mathbf{S B})-\arg (\mathbf{P}+\mathbf{C})$.

Note that in Eq. (3), we assume that the modulus of the coronagraphic pupil is never zero. However, formally speaking, one could wish to have an SB function that tends to zero at exactly the same locations as the zeros of the coronagraphic pupil complex amplitude, but this is an ill-posed situation and probably not realistic. Another important point to take into account are modulus and phase discontinuities along the $\mathrm{CO}$ and SA edges. This point is discussed in Sect. 5.1, but before that we consider the theoretical case where we can build a perfect complex amplitude filter. Finally, we also point out that the SB function is only defined in the pupil area and is therefore also acting as a pupil stop. Since a fraction of light is generally rejected outside this area due to the previous focal mask, this stop is blocking this light fraction and thus acting as a Lyot stop. This is why this first beam-shaping stage can be assimilated to a first coronagraphic stage.

\subsection{Filter throughput and global throughput}

We define the throughput as the amount of light transmitted by the system for an off-axis object. Talking about throughput for the on-axis source is not relevant here because our goal is to suppress it: on-axis throughput is just the coronagraph efficiency. Thus, the throughput of filter $\mathbf{F}$ only is obtained as

$T=\frac{\int\left|\mathbf{P}_{\text {geom }} \times \mathbf{F}\right|^{2}}{\int\left|\mathbf{P}_{\text {geom }}\right|^{2}}$

where $\mathbf{P}_{\text {geom }}$ is the geometric pupil defined as taking the value 1 everywhere except at the $\mathrm{CO}$ and SA locations (where it is zero). In that case we can write $\mathbf{P}=\mathbf{P}_{\text {geom }} \times \mathbf{W}$ where $\mathbf{W}$ can be an arbitrary function (e.g. an apodization function, or taking the value 1 everywhere).

We can also define a global throughput $T_{\text {global }}$ that accounts for the throughput for an off-axis object. It is computed as

$T_{\text {global }}=\frac{\int|\mathbf{P} \times \mathbf{F}|^{2}}{\int\left|\mathbf{P}_{\text {geom }}\right|^{2}}$.

There, $T$ and $T_{\text {global }}$ do not depend on the CO and SA area because an off-axis object is (almost) unaffected by the diffracting mask, as almost no light is diffracted inside the CO and SA structures and it is not affected by the beam-shaping filter. Also see Sect. 5.3 for a study of the limits where this statement still remains valid.

Note that in the case where $\mathbf{P} \neq \mathbf{P}_{\text {geom }}, T_{\text {global }}$ is not the simple product of the pupil throughput by the filter throughput since in general, both can be different functions. Moreover, $T_{\text {global }}$ is not even equal to the throughput of the output pupil (i.e. the SB function). The reason is that the filter is NOT the output pupil function. The case where $\mathbf{P} \neq \mathbf{P}_{\text {geom }}$ will not be addressed in this paper, but preliminary studies show that it can lead to interesting properties. Examples of possible throughput values are given in Sect. 4.1.

\section{Beam shaping with Lyot occulting masks and circular phase masks}

Among many expected possibilities, we wish to study two straightforward kinds of masks for the beam-shaping stage. We consider simple Lyot occulting mask first, then circular phaseshifting masks. In the following sections we use an arbitrary pupil geometry with a CO diameter that is $20 \%$ of the overall pupil diameter. The SA overall width is $3.75 \%$ of the pupil diameter (just for convenience in numerical simulations).

\subsection{Lyot occulting mask}

We start by studying the simple and arbitrary case where the output beam-shaped pupil is a flat uniform function (flat wavefront). In this case the desired beam shape SB function is defined simply as $\mathbf{S B}=1$ on the full circular pupil. According to Eqs. (4) and (5) the modulus and phase of $\mathbf{F}$ are

$|\mathbf{F}|=\frac{1}{\max \left(\frac{1}{|\mathbf{P}+\mathbf{C}|}\right)} \frac{1}{|\mathbf{P}+\mathbf{C}|}$

and, if we take $\varphi_{\mathbf{S B}}=0$,

$\varphi_{\mathbf{F}}=-\arg (\mathbf{P}+\mathbf{C})$.

As mentioned previously, Eq. (8) requires that the coronagraphic pupil modulus is never zero, which would lead to the trivial case $\mathbf{F} \equiv 0$. Figure 3 summarizes the principle of the beam-shaping operation in the pupil plane for this configuration. For the sake of simplicity, only the moduli are shown. More details about modulus and phase are given hereafter. 


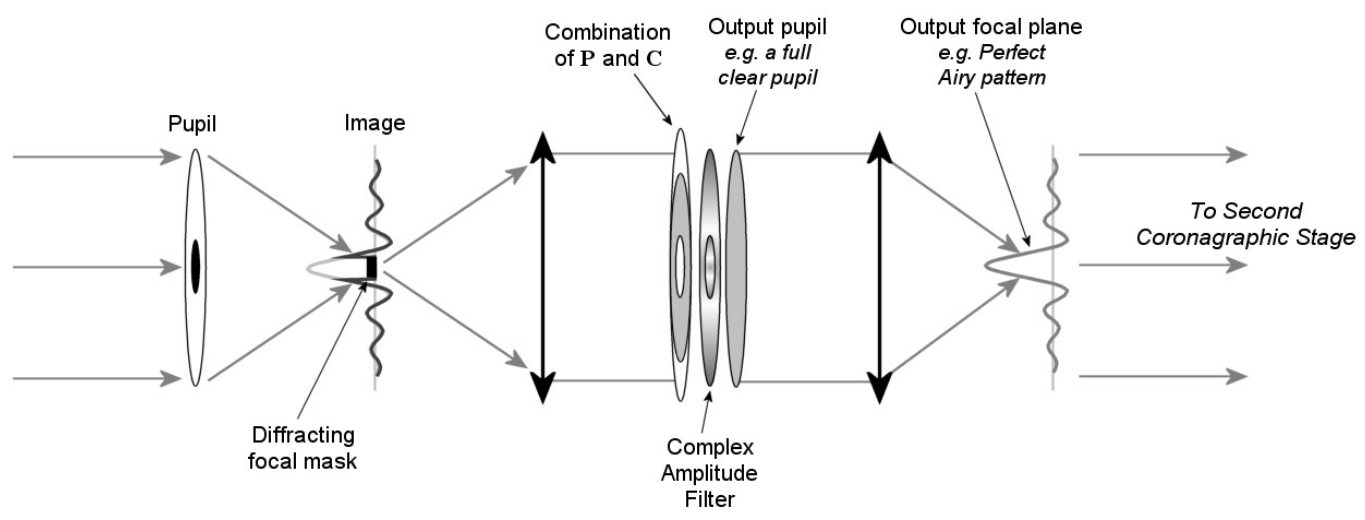

Fig. 2. Schematic of the beam-shaping coronagraphy. The diffraction effect of a focal mask in the re-imaged pupil naturally fills the CO and SA structures with coherent light. A special complex amplitude filter is placed in this pupil in order to shape the beam as needed for a downstream coronagraph.
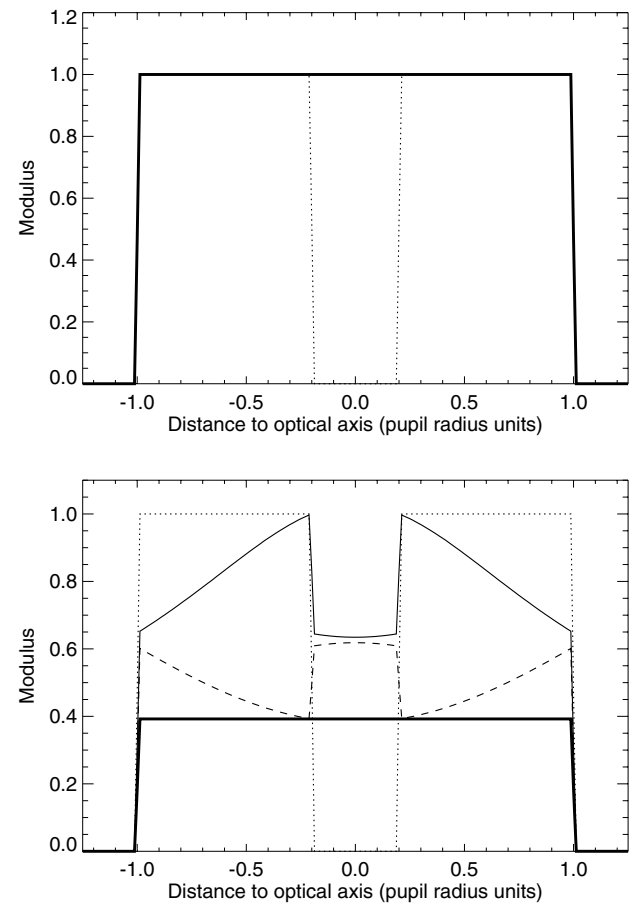

$$
T_{\text {global }} \approx 60 \%
$$

Fig. 3. Principle of the beam-shaping stage in the pupil plane. (Top) The entrance pupil (dotted) with a CO (and SA, not shown here) needs to be shaped to a flat output pupil (thick solid line, normalized) without CO and SA. (Bottom) After a diffracting mask is applied in the intermediate focal plane, the amplitude in the coronagraphic pupil plane (dashed) is multiplied to the filter $\mathbf{F}$ (solid line) in order to obtain the desired output pupil (thick solid line).

\subsubsection{Focal mask size and filter throughput}

Depending on the focal occulting mask size (size refers to the mask diameter), the diffracted part $\mathbf{C}$ will exhibit more or fewer "oscillations" (either in the CO and SA areas or in the unobscured part), and the final coronagraphic complex amplitude will have a more or less complex structure. Note that these fluctuations also depend on the entrance pupil geometry, especially the CO size, because part $\mathbf{C}$ also depends on the original pointspread function. But in the following, we only consider a given pupil geometry (defined above) and find ways to remove its $\mathrm{CO}$ and SA.

For the sake of simplicity in the graphical representation of the complex amplitude in the coronagraphic pupil plane $(\mathbf{P}+\mathbf{C})$ of Figs. 4, 5, and later in this paper, we have voluntarily chosen a real and symmetric entrance pupil, so that the imaginary part is zero for $\mathbf{P}+\mathbf{C}$, and the phase of the beam-shaping filter is in general $\pi$ (corresponding to a simple sign switch of the real part). However, the method remains completely applicable to the general case where the entrance pupil is asymmetric and/or complex. In that case, the coronagraphic pupil is also a complex function (see Eqs. (4) and (5) where no assumption is made on the phase or amplitude distributions) and the required complex amplitude filter needs to compensate for both phase (not necessary $\pi$ ) and amplitude.

Figures 4 and 5 show two examples of Lyot occulting mask used as a first diffracting stage with two different diameters $(1.2$ and $4.0 \lambda / D)$. These two cases correspond to a good and a bad solution, respectively: the small mask is the case where the modulus of the coronagraphic pupil never goes to zero and thus can be shaped and flattened. For the wider mask, the flattening cannot be achieved because the amplitude is zero at some locations in the unobscured part (see black areas top-left in Fig. 5). In the small mask case the filter $\mathbf{F}$ is computed according to Eqs. (8) and (9), while the modulus of $\mathbf{F}$ is shown in Fig. 6a.

Figure 7 shows a plot of the throughput against the variation of the diffracting Lyot mask size. The blank areas correspond to the case where the modulus in the coronagraphic pupil tends to zero (or actually goes to zero) at some locations, and where the corresponding throughput also tends toward zero (reminding the reader that we are in the specific case where we want to flatten the output complex amplitude). The plot exhibits two local throughput maxima ( $\approx 60 \% @ 1.2 \lambda / D$ and $\approx 43 \% @$ $3.2 \lambda / D$ ) revealing an optimal diffracting mask diameter. Note that in this example, the global throughput is identical to the filter throughput because $\mathbf{P}=\mathbf{P}_{\text {geom }}$. 

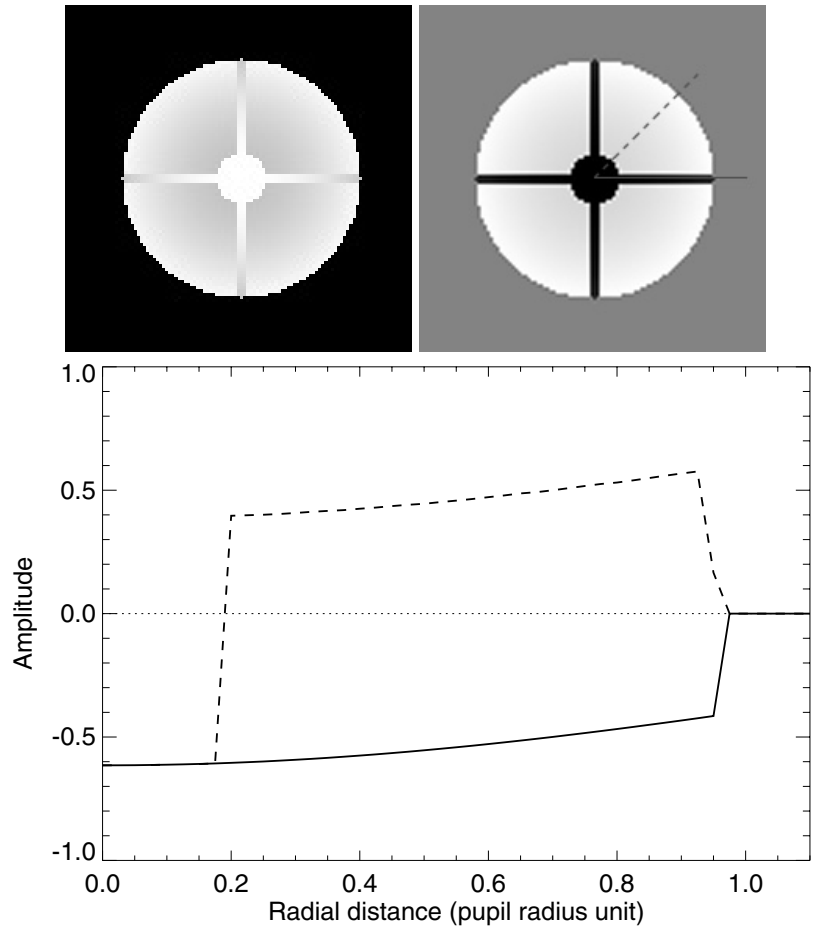

Fig. 4. (Top-left) Coronagraphic pupil modulus for a classical Lyot

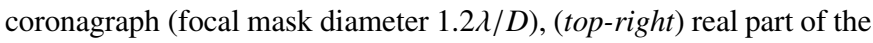
coronagraphic pupil complex amplitude $\mathbf{P}+\mathbf{C}$ (the imaginary part is null). The gray external area at top-right is zero, darker are negative values, and brighter are positive values. The modulus map is shown with a power-law display (not linear). (Bottom) Cuts of the real part of the 2D coronagraphic pupil amplitude (top-right) along the horizontal axis (continuous line) and along $45^{\circ}$ (dashed line) representing, respectively, the amplitude in the $\mathrm{CO} / \mathrm{SA}$ and the amplitude in the unobscured part.

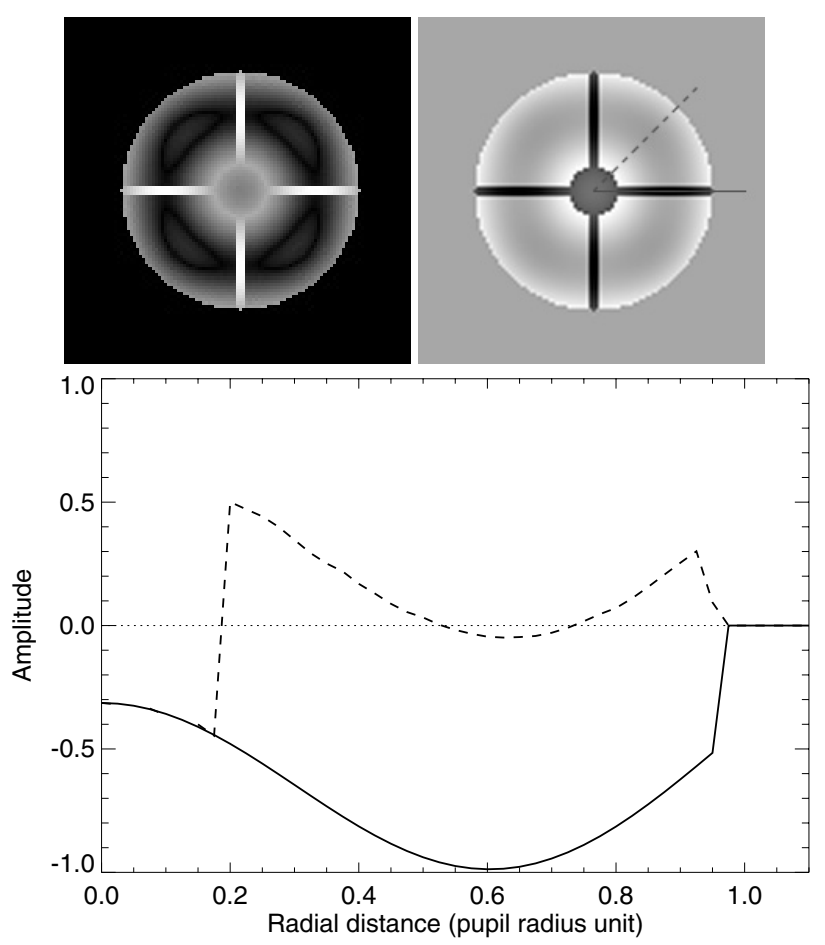

Fig. 5. Same as Fig. 4 but for a focal mask diameter of $4.0 \lambda / D$.

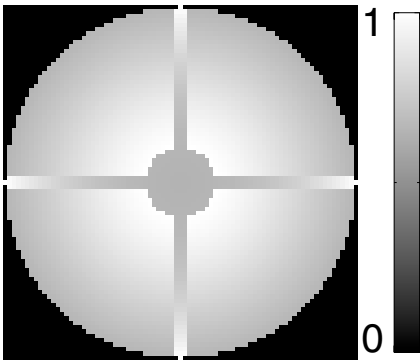

(a)
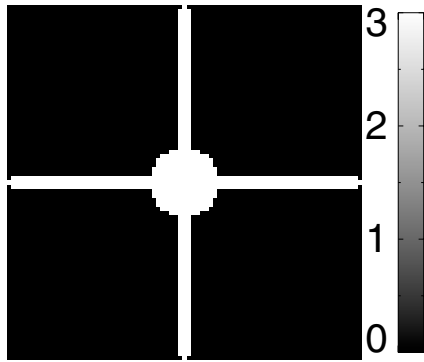

(b)
Fig. 6. Modulus a) and phase (radians, b)) of the beam-shaping filter $\mathbf{F}$ in the conditions of Fig. 4.

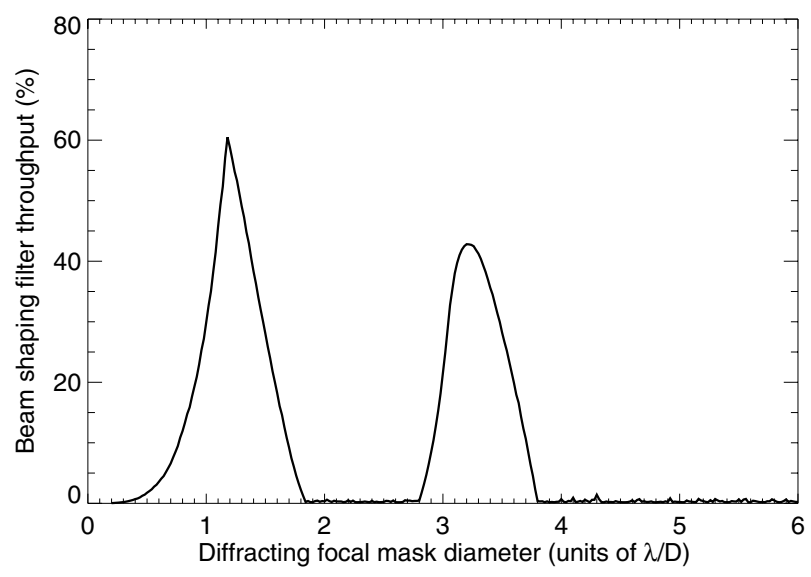

Fig. 7. Throughput $T$ (Eq. (6)) of the beam-shaping filter $\mathbf{F}$ versus diffracting focal mask diameter for the pupil configuration described in Sect. 4.1. Small fluctuations in between the two peaks should tend toward zero and are due to the numerical sampling of the pupil that cannot correctly sample the zeros of the modulus.

\subsubsection{Filter phase map}

The Lyot occulting phase mask function $\mathbf{M}$ can be written as $\mathbf{M}=1-\Pi_{a}$ (Aime et al. 2001) where $\Pi_{a}$ is the top-hat function taking a value of unity on a disk of radius $a$ and zero elsewhere. In the focal mask plane and with the Fourier optics approximation, the complex amplitude can be written,

$\mathbf{A}_{\mathrm{cor}}=\mathrm{FT}(\mathbf{P}) \times \mathbf{M}=\mathrm{FT}(\mathbf{P}) \times\left(1-\Pi_{a}\right)$

where FT( $\cdot)$ denote the Fourier transformation. The conjugate (coronagraphic) pupil $\mathbf{P}_{\text {cor }}=\mathrm{FT}\left(\mathbf{A}_{\text {cor }}\right)$ is

$\mathbf{P}_{\mathrm{cor}}=\mathbf{P} *\left(\delta-\mathrm{FT}\left(\Pi_{a}\right)\right)=\mathbf{P}-\mathbf{P} * \mathrm{FT}\left(\Pi_{a}\right)$.

The rightmost negative convolution product is similar to the term $\mathbf{C}$ defined previously. In the case of a perfectly flat entrance wave-front, the imaginary part of the complex amplitude $\mathbf{P}_{\text {cor }}$ is null and only remains the real part. We can thus analyze the phase map by looking at the complex amplitude (real part) sign. Provided the mask size is not too large, the convolution product (Eq. (11)) is a heavily smoothed version of the entrance pupil with no "oscillations" (as mentioned in Sect. 4.1.1). This convolution product can never have a higher modulus than the unobscured part of the entrance pupil function $\mathbf{P}$, because the fraction of energy encompassed by the mask 
function $\mathbf{M}$ is trivially always lower than the overall energy. Therefore the sign of $\mathbf{P}_{\text {cor }}$ is expected to be mostly positive everywhere in the unobscured part. On the contrary, the sign of the amplitude pupil function inside the $\mathrm{CO}$ and SA will always be negative (i.e. a relative $\pi$ phase-shift) because $\mathbf{P}$ is null at these locations. The phase map is shown in Fig. 6b where it is $\pi$ at the $\mathrm{CO}$ and SA location and zero elsewhere.

\subsubsection{Interpretation and definition of ideal filters}

A balance develops at the throughput local maxima between the concentration of (diffracted) light in the $\mathrm{CO} / \mathrm{SA}$ patterns and the unobscured part. Also, the modulus fluctuations of the $\mathbf{P}+\mathbf{C}$ function are not too steep, either in the $\mathrm{CO} / \mathrm{SA}$ or in the unobscured part. As a consequence, the filter only needs to slightly modify this coronagraphic pupil amplitude function (but once again, in the particular case of a flat output pupil).

This helps us understand what the optimal coronagraphic pupil (and therefore the optimal filter) should be for the beamshaping operation. First, we would like an amplitude function that would require a filter only affecting the $\mathrm{CO}$ and $\mathrm{SA}$, leaving the unobscured part as is. A consequence would be that the unobscured part function is as close as possible to the desired output pupil function. Second, and for the sake of coronagraphic performance, we would like the unobscured part to be as low as possible with a maximum concentration of diffracted light in the CO and SA (i.e. a high efficiency of the first diffracting - coronagraphic - mask). We are currently exploring this possibility in the case of amplitude apodized entrance pupils. A general study of these optimal cases is not really the goal of this paper, so we leave it to a forthcoming paper. In this paper we want to focus on what appears to be much simpler and straightforward cases. Finally, and for the sake of simplicity, we would like to have a filter that only works in modulus. This feature can be obtained under certain conditions, as explained in the next section.

\subsection{Circular phase mask}

In principle, coronagraphic phase mask should refer to the Roddier \& Roddier (1997) coronagraph. In the present case we want to study the use of a phase-shifting mask for the beamshaping operation. As explained in Aime et al. (2001), and similarly to the equations of Sect. 4.1.2, the phase mask function $\mathbf{M}$ can be written as $\mathbf{M}=1-2 \Pi_{a}$. The focal plane amplitude function at the $\pi$ phase-shifting mask location is, in the Fourier optics approximation

$\mathbf{A}_{\mathrm{cor}}=\mathrm{FT}(\mathbf{P}) \times\left(1-2 \Pi_{a}\right)$.

Thus the conjugate (coronagraphic) pupil $\mathbf{P}_{\text {cor }}$ can be written

$\mathbf{P}_{\text {cor }}=\mathbf{P} *\left(\delta-2 \mathrm{FT}\left(\Pi_{a}\right)\right)=\mathbf{P}-2 \mathbf{P} * \mathrm{FT}\left(\Pi_{a}\right)$.

Again, the rightmost negative convolution product is similar to the term $\mathbf{C}$ defined previously.

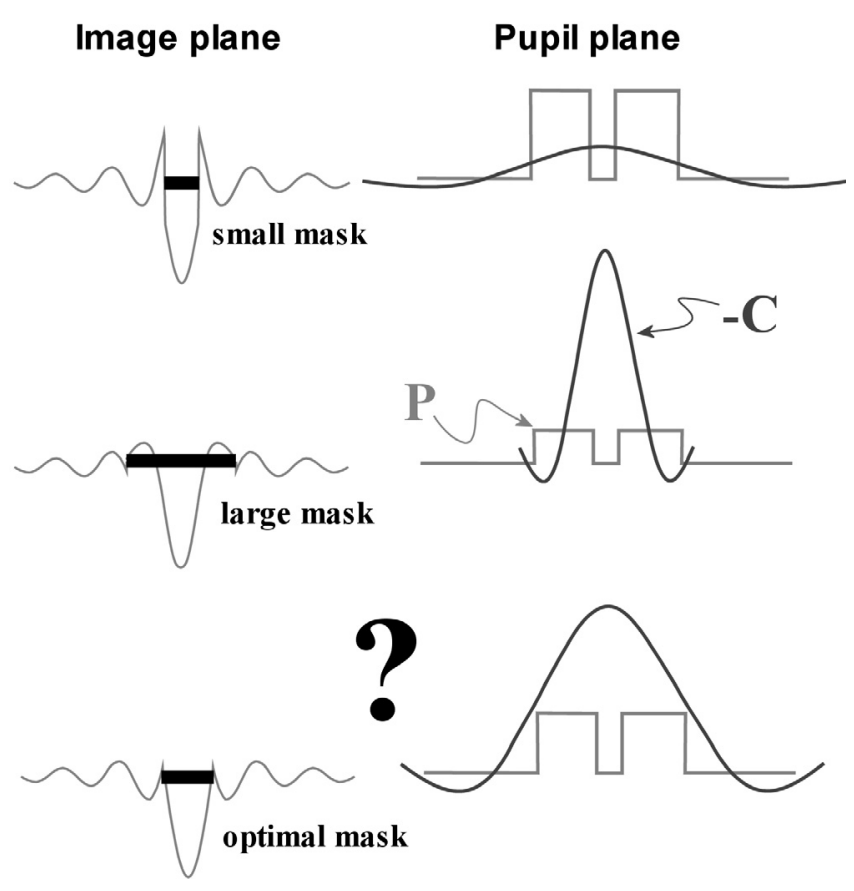

Fig. 8. (Top) A small mask produces a wide and low amplitude term $\mathbf{C}$ compared to $\mathbf{P}$, so $\mathbf{P}+\mathbf{C}$ has a sign switch at the $\mathrm{CO} / \mathrm{SA}$ location. (Middle) A very large mask produces a narrow and high amplitude term $\mathbf{C}$ compared to $\mathbf{P}$, but $\mathbf{P}+\mathbf{C}$ falls to zero somewhere inside the geometric pupil. (Bottom) Can we find an intermediate mask size which produces a term $\mathbf{C}$ whose amplitude is higher than $\mathrm{P}$ everywhere in the geometric pupil?

\subsubsection{Basic case}

As in the Lyot case, the focal phase mask size will control the equivalent width of the term $\mathbf{C}$, as well as its relative amplitude compared to $\mathbf{P}$. Since the convolution product in Eq. (13) is multiplied by a factor 2 , we can expect that for a certain mask size, the term $\mathbf{C}$ amplitude will be larger than $\mathbf{P}$ everywhere in the pupil. In that case, the sum $\mathbf{P}+\mathbf{C}$ will have a constant (negative) sign everywhere in the pupil and the filter $\mathbf{F}$ will only need to work in modulus and not in phase. If the mask size is too small, the amplitude of $\mathbf{C}$ will not be high enough to dominate the term $\mathbf{P}$ and sign switches ( $\pi$ phase-shifts) will occur. And if the mask is too large, $\mathbf{C}$ will have a high amplitude, but will become narrow and start to produce zero amplitude points inside the pupil, preventing construction of the beamshaping filter. These different situations are depicted in Fig. 8. It is important to mention that this "non phase-shifting" case can only be obtained when the entrance pupil is symmetric and real, so that the imaginary part is zero for the $\mathbf{P}+\mathbf{C}$ term. This would therefore require an additional geometric pupil mask in the case of a non centro-symmetric entrance pupil.

In order to find the optimal mask size and see if this balance can be found, we computed the filter throughput and checked if the filter needed a phase-shift at the CO and SA locations. This is summarized in Fig. 9 (top-left). We can see that there is no solution for this given pupil geometry, so the optimal beam-shaping filter also requires a $\pi$ phase-shift in the $\mathrm{CO}$ and SA pattern. Note that the maximum throughput for the phase-shifting solution is higher $(\approx 73 \%)$ than for the Lyot case 

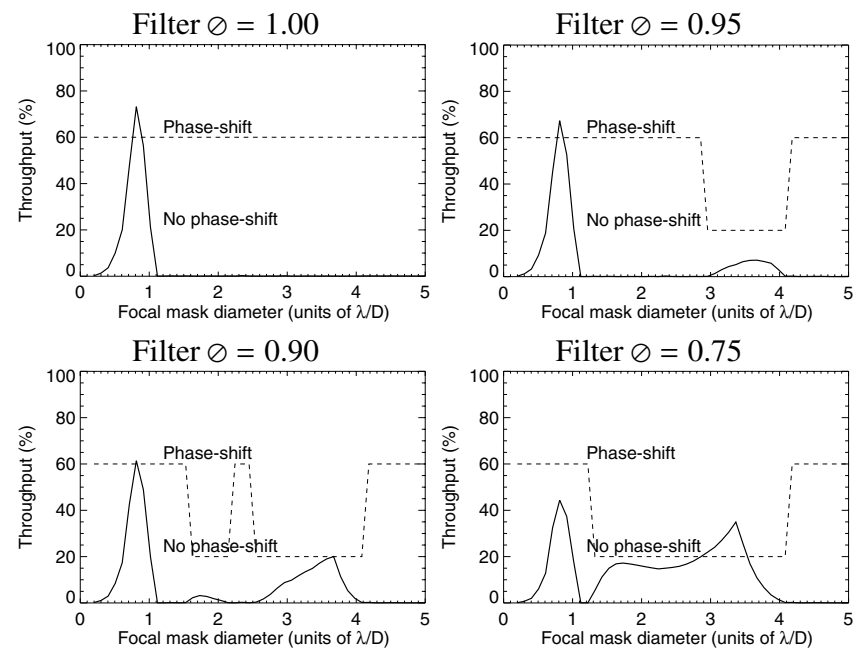

Fig. 9. Throughput and need for filter phase-shift compared to the focal phase-shifting mask diameter. Figures depict four cases with decreasing filter effective diameter: full pupil (top-left), 95\% (top-right), 90\% (bottom-left) and 75\% (top-right), the latter being close to optimal throughput. The dashed line indicates the need for a filter phaseshift (upper value) or not (lower value).

$(\approx 60 \%)$. We also found out, as expected from the discussion above, that the zeros of the coronagraphic pupil function are appearing at the outer edges of the pupil as the focal mask size increases. Thus, if the filter's size is slightly reduced, we can find a continuous set of solutions where no phase shift occurs and where the throughput is not null (see Fig. 9). Still, the throughput for these solutions is not very high, and the effective achievable angular resolution is decreased. Nevertheless, the optimal throughput for the non phase-shifting solutions is about $35 \%$ (Lyot stop diameter $75 \%$ ), which can still be considered as reasonable if we compare it, for example, to a PALC (Soummer et al. 2003).

\subsubsection{Influence of pupil geometry and apodization}

Without going too much into detail, we state here again that the mentioned performance strongly depends on the $\mathrm{CO}$ and SA geometry. Changing the size of the $\mathrm{CO}$ or SA or both leads to significantly different results in the behavior of the filter throughput along with the focal mask size. Therefore, and depending on the cases, we can envisage that the pupil geometry can be slightly modified prior to entering the beamshaping stage in order to optimize the throughput, and in a more general way, to match the constraints of the optical setup (e.g. manufacturing constraints). We are also investigating the possibility of using amplitude-apodized entrance pupils in combination with circular phase-shifting focal masks. This combination should allow the use of a full diameter Lyot stop while preserving optimal throughput, but we leave this study to a future paper.

\section{Practical implementation}

Theoretically speaking, a perfect flat wavefront of an onaxis point source can be obtained by the beam-shaping coronagraphy when a solution is found for a given pupil geometry. Then an ideal coronagraph can be applied in the next stage. In the real world, degradations will occur because of system-tuning errors and defects in optical components manufacture. In this section, we try to examine the possible degradation effects that are due to the complex amplitude filter manufacturing and to pointing errors.

\subsection{Filter discontinuities}

The pupil-filter discontinuities in both phase and amplitudes lead to the non uniformity of the output pupil. However we can envisage that the scale of these transition areas can be made very small compared to the scale of the pupil. It seems reasonable to envisage that the complex amplitude filter pattern can be fabricated with a precision on the order of a few tens of microns (e.g. $50 \mu \mathrm{m}$ ) in both amplitude and phase. For a $10 \mathrm{~mm}$ re-imaged pupil size, this corresponds to $0.5 \%$ of the pupil diameter.

We tried to evaluate the impact on performance of these manufacturing defects. In our simulations, the discontinuities are made opaque; this masking can be obtained by a high optical density in the modulus term of the filter. The beam is flattened using a Lyot occulting beam-shaping stage (Sect. 4.1) while the downstream coronagraphic stage is chosen to be a FQPM coronagraph because of its sensitivity to the CO. Figure 10 shows the pupil geometry (left column) and the corresponding masking of the discontinuities (right column) in two pupil shape cases: $\mathrm{CO}$ and cross-shaped SA, and a Subaru/VLT-like CO/SA structure. Figure 11 shows the (second stage) coronagraphic pupil in the case of a FQPM coronagraph used in combination with a flat beam-shaped output pupil. Figure 12 shows the azimuth-averaged profiles in the final coronagraphic image for the two mentioned pupil shapes. The gain is largely improved by a factor $50 \sim 100$ with the beam-shaping configuration. Table 1 gives the throughput values and the optimal beam-shaping mask size for the above configurations.

There is no significant throughput difference with and without the beam-shaping stage, although it is slightly lower in the beam-shaped case. The Lyot stop in the beam-shaping case is just a slightly reduced version of the entrance pupil $(98 \%$ of effective diameter) including the CO and SA. In the standard case, the Lyot stop is computed using a threshold method as proposed by Riaud (2001).

\subsection{Filter phase-shift}

The phase-shifts we used in this study were mostly pure $\pi$ phase-shifts. However, in the real world, some phase fluctuations should arise from the imperfections of the setup (e.g. asymmetry of the entrance pupil). We have not yet investigated the possibilities of actually performing such "local phaseshifts" from a technical point of view, although it seems possible to use dielectric coatings to reproduce some defined phase map with very good precision (INO, private communication). Another possibility for setting the filter phase map is to use 

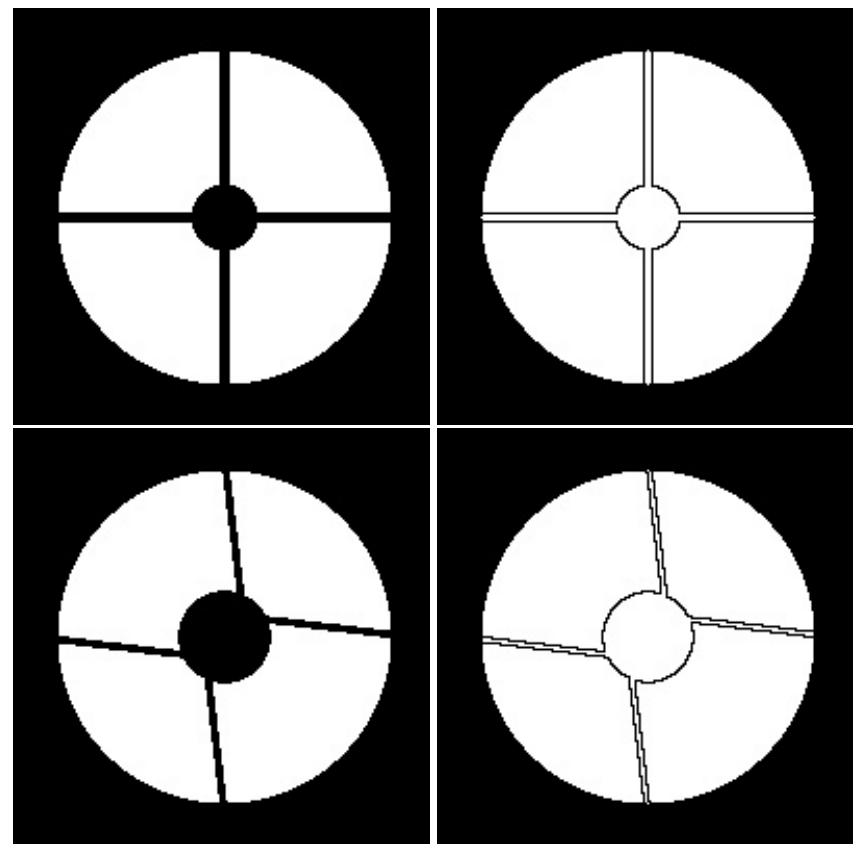

Fig. 10. Masking of discontinuity edges. (Left) The entrance pupil shape and the output beam-shaped pupil (right). The top row is the cross-shaped SA, and the bottom row is the Subaru/VLT-like SA structure.

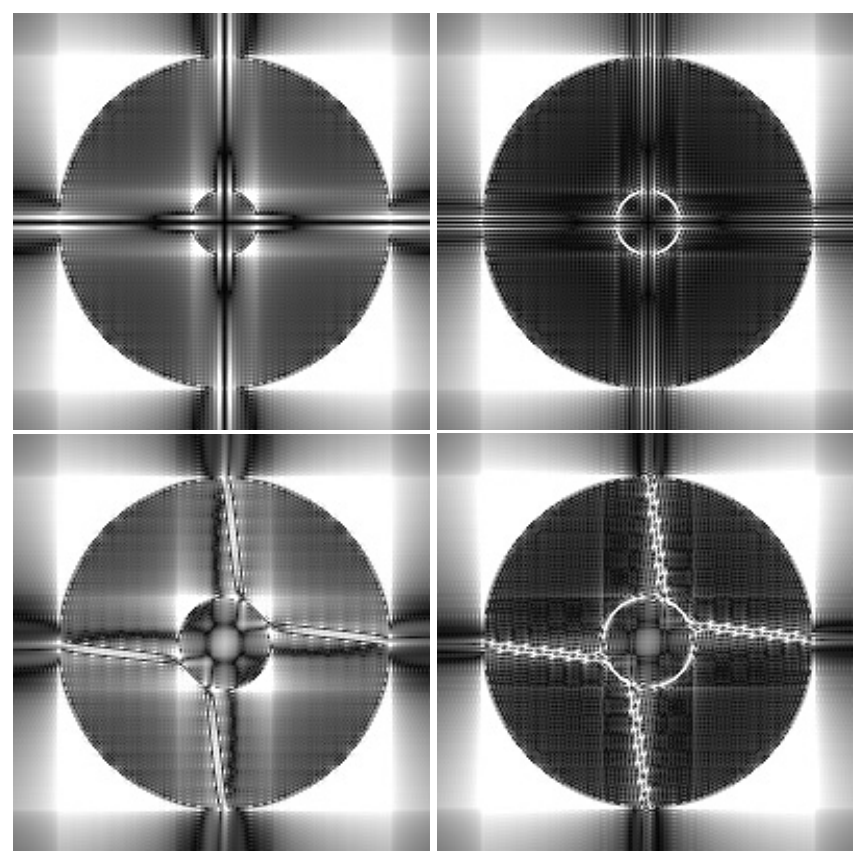

Fig. 11. Second coronagraphic pupil for the FQPM coronagraph without beam-shaping (left) and with beam-shaping (right). Images are displayed as a power 0.3 of intensity.

deformable mirrors with a high density of actuators (e.g. MEM DM). In that case, the use of asymmetrical pupils, eventually including known phase defects, could be manufactured and provide the desired phase filter. Finally we may be facing two difficulties that could be technically incompatible for manufacturing a single optical component: the manufacturing of a spatially variable phase map for correcting low amplitude phase
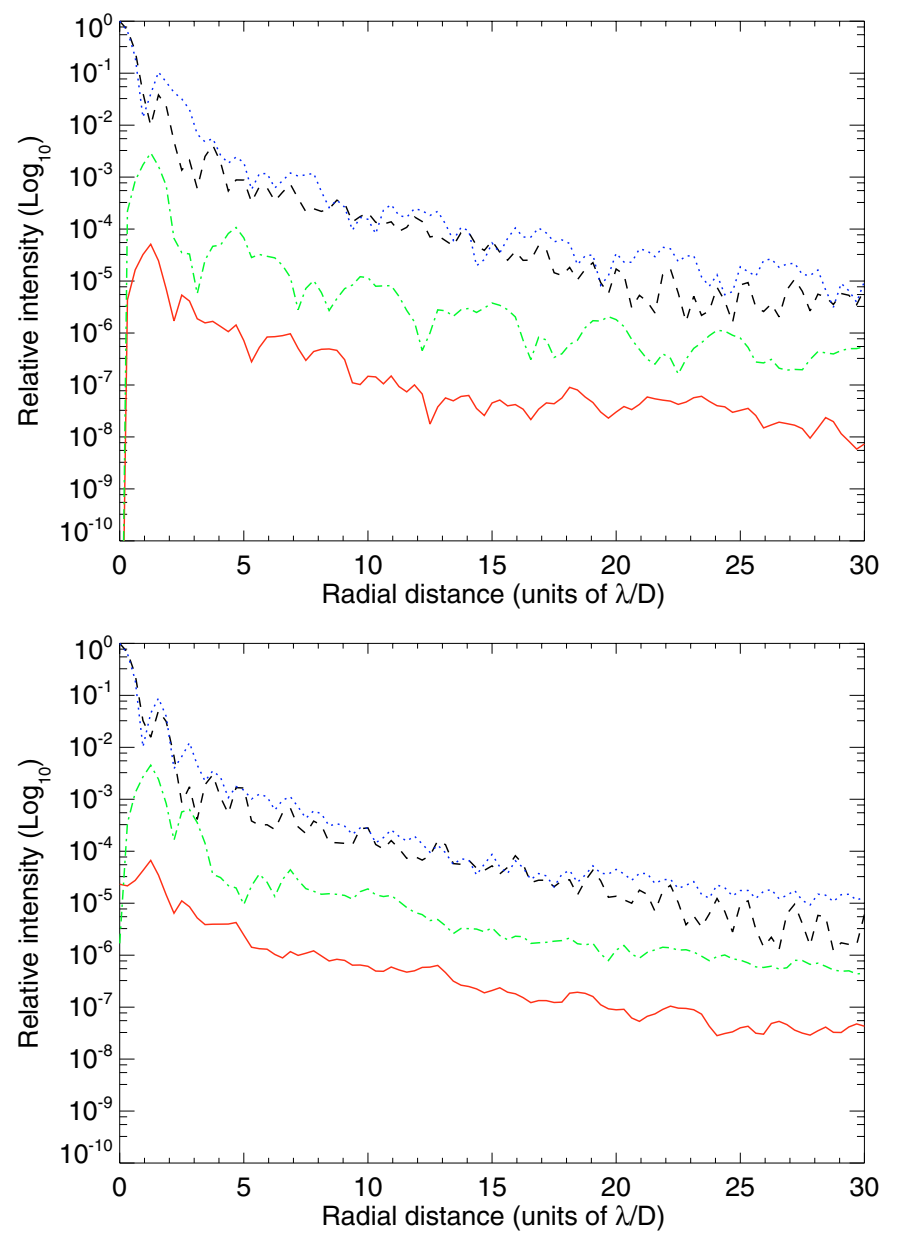

Fig. 12. Performance comparison with an FQPM coronagraph with and without beam shaping (azimuth averaged profiles). (Top) corresponds to a CO with a cross-shaped SA. (Bottom) corresponds to a Subaru/VLT-like pupil shape. In both figures, the curves correspond to the reference FQPM image without coronagraphic mask but with Lyot stop and without beam-shaping (dotted), the coronagraphic FQPM image without beam-shaping (dash-dotted), the reference FQPM image without coronagraphic mask but with Lyot stop and with beamshaping (dashed), the coronagraphic FQPM image with beam-shaping (continuous). The performance with beam-shaping is normalized to the second stage Lyot stop transmission multiplied by the BS filter transmission.

Table 1. Simulation parameters for a flat output pupil (shaped beam) and with a second stage FQPM coronagraph. The Lyot stop used for the FQPM without beam shaping (BS) have been tuned with an energy threshold method. However, in practice, this Lyot stop may need further tuning, probably with a slightly lower throughput.

\begin{tabular}{lcc}
\hline \hline Parameter & Cross-shaped & Subaru/VLT \\
\hline Global Transmission w/o BS & $57 \%$ & $72 \%$ \\
Global Transmission w/ BS & $62 \%$ & $61 \%$ \\
BS focal mask diameter & $1.30 \lambda / D$ & $1.48 \lambda / D$ \\
\hline
\end{tabular}

errors, and the possibility of including sharp and steep phase changes (necessary for the Lyot mask case). However as explained above, the sharp transition areas problems can be partly 


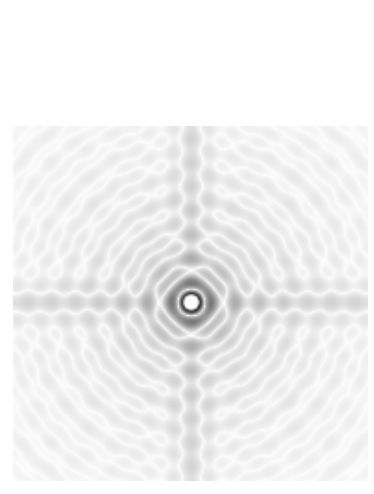

(a)
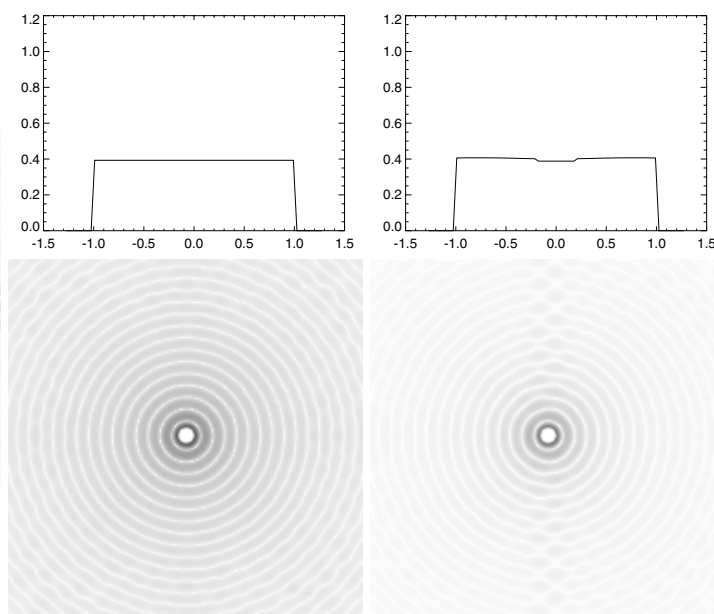

(b)

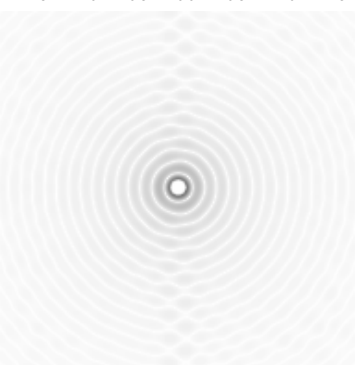

(c)
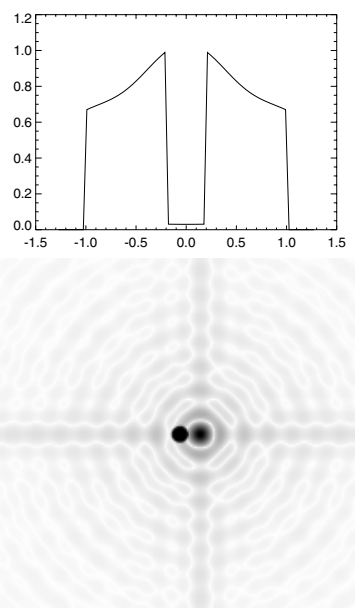

(d)

Fig. 13. Effect of the off-axis point source position on the output pupil and corresponding focal image, in the configuration described in Sect. 4.1 with the small $1.2 \lambda / D$ mask. a) Prime focus image with the diffracting focal mask superimposed (white central disk); it corresponds to the plane denoted image in Fig. 2. From b) to d), the top line represents diagonal cuts in the output pupil modulus, while the bottom line shows the corresponding focal plane images (FT of the output pupil). The input tip-tilt amplitudes and the final peak-to-peak attenuation (in percent) compared to the image in a) are from b) to d): $0.0 \lambda / D(19.7 \%), 0.1 \lambda / D(20.4 \%)$, and $2.0 \lambda / D(57.7 \%)$, respectively. The top line shows the re-apparition of discontinuities at the CO and SA locations, resulting in SA diffraction patterns in the focal plane images of the bottom line. Images are shown with a 0.3 power exponent. On image d), the occulting spot is shown in black for display convenience.

overcome by masking them with the amplitude part of the complex filter.

\subsection{Off-axis objects and pointing errors}

As suggested before, an off-axis object will be affected by the first stage focal mask in proportion to its off-axis distance. Nonetheless, the distance beyond which the first mask effect is significant is at most $\sim \lambda / D$ from the edge of the focal mask (but of course it depends on the type of focal mask used). An additional effect is the quick re-apparition of the SA diffraction pattern since no light (or a negligible fraction) is present in the CO and SA patterns in the coronagraphic pupil plane. The modulus and phase of the complex amplitude for this off-axis object are, however, modified by the filter $\mathbf{F}$ complex function. But in the example given in the previous section, we pointed out that the phase of the filter only affects the SA and SO structures. Therefore, the off-axis object's complex amplitude will (almost) only be modified in modulus. Figure 13 (bottom line) shows off-axis images in the output focal plane (see Fig. 2) in the configuration described in Sect. 4.1 with the small $1.2 \lambda / D$ mask.

Different radial distances were considered. For comparison, the leftmost image corresponds to the prime focus image (image plane of Fig. 2). Recalling that the global throughput of the filter $\mathbf{F}$ is $\sim 61 \%$, the indicated attenuations of the peak images suggest the attenuation effect of the coronagraphic mask at small distances and the small effect of the modification of the amplitude function through the complex filter at large distances. The top line of this figure shows diagonal cuts (no cut through the SA) of the pupil modulus where we can see the apparition of discontinuities at the $\mathrm{CO}$ and SA locations, creating diffraction spikes in the focal image plane.

The case of pointing accuracy can be treated in the same manner as the off-axis object. The question remains if a second stage coronagraph would see its performance altered for a pointing error of a given amplitude. Figure 14 shows the case where a FQPM coronagraph is used (as the second stage coronagraph in the case of the beam-shaping configuration) and where a tip-tilt is included (amplitude of $0.1 \lambda / D$ at an azimuth of $45^{\circ}$ ). The relative performance degradation with the beam-shaping configuration is higher when compared to the perfectly on-axis source (Fig. 12), but still the global performance is higher than using the FQPM alone. This means that the beam-shaping still provides a good quality downstream wavefront in both amplitude and phase to feed a FQPM coronagraph, which is known to be already rather sensitive to pointing errors. Further studies should assess the sensitivity of several other coronagraphs to this wavefront degradation.

\section{Summary and discussion}

We presented a generic principle that allows the removal of $\mathrm{CO}$ and SA for a perfectly on-axis source. It relies on a natural coherent "filling" of the inner pupil geometry (i.e. CO and SA) by the use of a first diffracting component (a focal mask in this case) and an associated complex amplitude pupil filter (modulus and phase). The reshaped pupil then serves as an input into a coronagraph (or whatever is needed to remove the $\mathrm{CO}$ and SA for an on-axis source). We mainly analyzed the effect of the focal mask size in the case where an occulting spot is used as a diffracting mask, and showed that an optimal mask diameter can maximize the complex amplitude filter throughput. By 

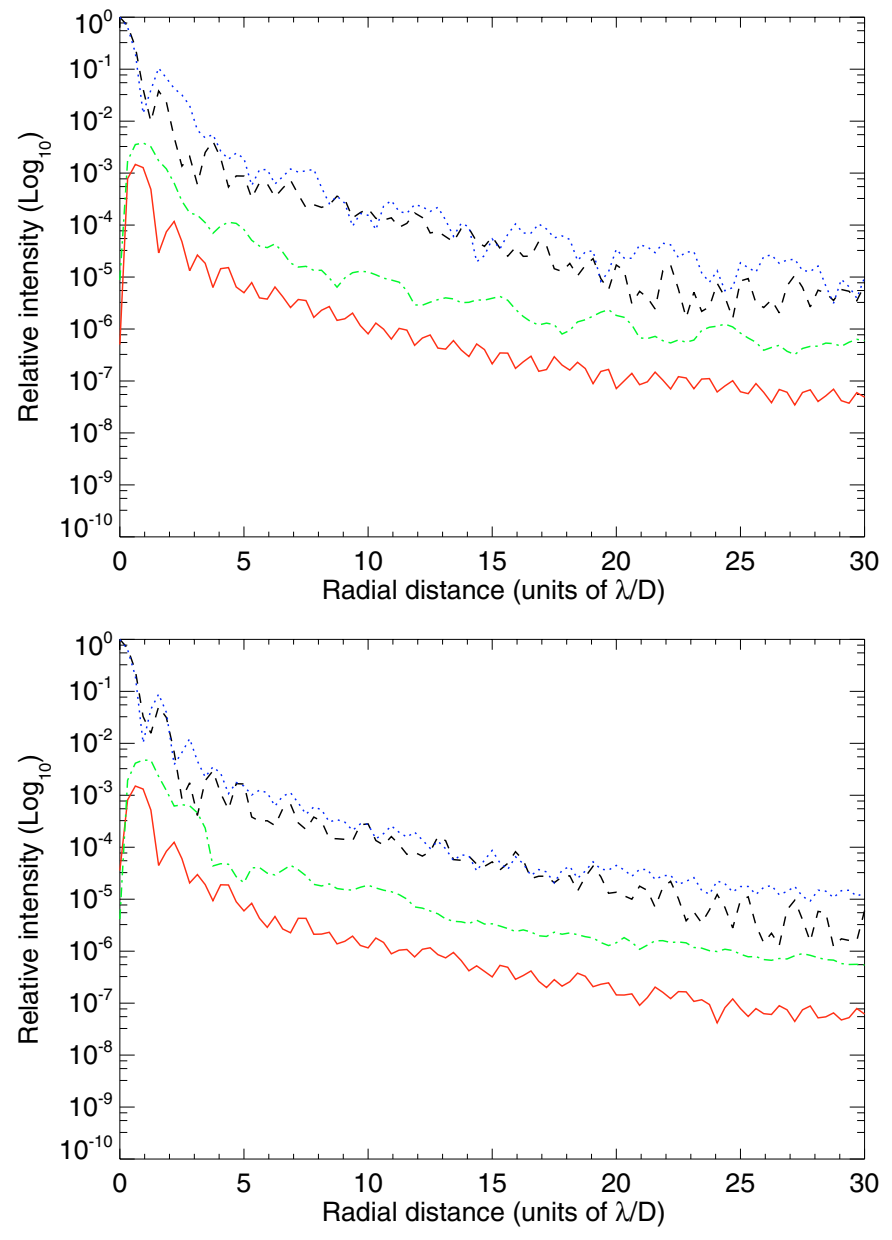

Fig. 14. Performance comparison of the beam-shaping with an FQPM coronagraph with and without beam shaping and for a tip-tilt of $0.1 \lambda / D$ amplitude at $45^{\circ}$. (Top) corresponds to a CO with a crossshaped SA. (Bottom) corresponds to a Subaru/VLT-like pupil shape. See Fig. 12 for a description of the curves.

using a circular $\pi$ phase-shifting mask, we showed that it is possible to use a complex amplitude filter that does not need to work in phase, but only in modulus. However this requires a slight reduction of the output pupil diameter, and thus lowers the throughput. Finally, we conclude that the beam-shaping optimization is not a straightforward operation and strictly depends on the design of the original pupil geometry.

Practical implementation can be difficult because of the discontinuities between the $\mathrm{CO} / \mathrm{SA}$ and the unobscured part, especially for the phase shifts. One possibility could consist in apodizing the $\mathrm{CO}$ and SA edges in phase and/or amplitude so that a smooth transition can occur, but such a study is beyond the scope of this paper. Nevertheless, current technology allows the manufacture of precise and relatively complex amplitude filters. For example, HEBS-glass technology can be used to produce high-fidelity modulus filters in the visible and nIR. The phase correction can be performed by suitable MEM deformable mirror or liquid crystal light modulators (e.g. Littman et al. 2003).
The other important point concerns the chromatism of the presented method. Although the proposed beam shaping relies on chromatic components (e.g. focal occulting mask), it may be possible to use additional optics (e.g. achromatic corrector by Wynne 1979). More sophisticated technology for achromatic phase masks using multi-material phase shift (Winsor et al. 2005) or thin films technology (Lemarquis \& Riaud 2003) could also solve some of the chromaticity problems. However, preliminary simulations seem to indicate that the technique is not too sensitive to chromatism: this could be due to the facts that the first beam-shaping stage already acts as a coronagraph and that the output pupil is still uniform enough (i.e. with a strong attenuation of the pupil geometry effects) for the downstream coronagraph. But once again, the proposed technique is meant to be a generic method for removing $\mathrm{CO}$ and SA, so more advanced solutions need to be found to accommodate these difficulties (e.g. more complex diffracting masks or entrance pupil pre-shaping). We also note that not all diffracting masks are suitable for the beamshaping technique. For example, the FQPM does not allow the diffracted light to fill the $\mathrm{CO}$ and SA: see for example Murakami \& Baba (2005) for an illustration of the CO effect in the coronagraphic pupil. In a general frame, this beam shaping technique can be applied to other modes than coronagraphy, where the spider diffraction pattern for on-axis sources is a source of image quality degradation. The progress of adaptive optics systems providing high Strehl ratios will soon become a reality. In that context, telescopes should be allowed to use advanced coronagraphic techniques. The present method could be a solution for overcoming (or at least minimizing) the pupil geometry problems and therefore could allow very high contrast imaging, especially from the ground.

Acknowledgements. LA and MT are supported by Grants-in-Aid (Nos. 160772048007 and 160871018002) from the Ministry of Education, Culture, Sports, Science, and Technology (MEXT) of Japan. The authors wish to thank K. Enya, T. Nakagawa, and the SPICA coronagraph team for fruitful and helpful discussions.

\section{References}

Abe, L., Boccaletti, A., \& Vakili, F. 2001, A\&A, 374, 1161

Aime, C., \& Soummer, R. 2003, SPIE Proc., 5490, 456A

Aime, C., Soummer, R., \& Ferrari, A. 2001, A\&A, 379, 697

Aime, C., Soummer, R., \& Gori, P.-M. 2003, EAS Pub., 8, 281A

Baba, N., \& Murakami, N. 2003, PASP, 115, 1363

Chakraborty, A., Thomson, L. A., \& Rogosky, M. 2005, Opt. Expr., $13,2394 \mathrm{C}$

Gay, J., \& Rabbia, Y. 1997, C. R. Acad. Sci. Paris, tome 332, Série II, 265

Guyon, O. 2003, A\&AS, 404, 379

Kasdin, N. J., Vanderbei, R. J., Spergel, D. N., \& Littman, M. G. 2003 , Proc. SPIE, 4860, 240

Kuchner, M. J., \& Traub, W. A. 2002, ApJ, 570, 900

Kuhn, J. R., \& Hawley, S. L. 1999, PASP, 111, 601

Labeyrie, A. 1999, ASP Conf, 194, 350L

Lemarquis, F., \& Riaud, P. 2003, Appl. Opt., 42, 34 
Littman, M. G., Carr, M., Leighton, J., et al. 2003, SPIE Proc., 4854, 405

Lyot, B. 1939, MNRAS, 99, 579

Nishikawa, J., Kotani, T., Murakami, N., et al. 2005, A\&A, 35, 379

Maylak, P. H. 1992, Appl. Opt., 31, 4377

Murakami, N., \& Baba, N. 2005, PASP, 117, Issue 829, 295-299

Riaud, P., Boccaletti, A., Rouan, D., Lemarquis, F., \& Labeyrie, A. 2001, PASP, 113, 1145

Roddier, F., \& Roddier, C. 1997, PASP, 109, 815

Rouan, D., Riaud, P., Boccaletti, A., Clénet, Y., \& Labeyrie, A. 2000, PASP, 112, 1479
Soummer, R., Aime, C., \& Fallon, P. 2003, A\&A, 397, 1161

Spergel, D. N., \& Kasdin, N. J. 2001, 99th AAS Meeting, 33, 1431

Tavrov, A., Bohr, R., Totzeck, M., Tiziani, H., \& Takeda, M. 2002, Optics Letter, 27, 2070

Traub, W. A., \& Vanderbei, R. J., 2003, ApJ, 599, 695

Vanderbei, R. J., Kasdin, N. J., \& Spergel, D. N. 2003, ApJ, 615, 555

Vanderbei, R. J., Kasdin, N. J., \& Spergel, D. N. 2004, ApJ, 590, 593

Winsor, R. 2005, SPIE Proc., 5487, 1402

Wynne, C. G. 1979, J. Opt. Comm., 28, 21 